\title{
Using a Steerable Projector and a Camera to Transform Surfaces into Interactive Displays
}

\author{
Claudio Pinhanez \\ IBM T.J. Watson Research Center \\ 30 Saw Mill River Road \\ Hawthorne, NY10532, USA \\ +1914 784-7009 \\ pinhanez@us.ibm.com
}

\begin{abstract}
The multi-surface interactive display projector (MSIDP) is a steerable projection system that transforms non-tethered surfaces into interactive displays. In an MSIDP, the display image is directed onto a surface by a rotating mirror. Oblique projection distortions are removed by a computergraphics reverse-distortion process and user interaction (pointing and clicking) is achieved by detecting hand movements with a video camera. The MSIDP is a generic input/output device to be used in applications that require computer access from different locations of a space or computer action in the real world (such as locating objects). In particular, it can also be used to provide computer access in public spaces and to people with locomotive disabilities.
\end{abstract}

\section{Keywords}

Ubiquitous computing; interaction devices; user interfaces.

\section{INTRODUCTION}

The Everywhere Displays project aims to develop systems that allow the transformation of any surface in a space into a projected "touch screen", without the need of installing LCD displays or of wiring sensors to the surface. We are currently developing a system that deflects the image projected by an LCD projector using a rotating mirror and senses user interaction with a video camera (see Figure 1). With this apparatus, we allow not only non-tethered access to computer displays from any surface of a space but also enable the computer to directly act upon objects and people in the real world through the projection of images and light patterns.

\section{THE MULTI-SURFACE DISPLAY PROJECTOR}

The basic device being used to create everywhere displays is the multi-surface display projector, or MSDP, composed of an LCD projector and a computer-controlled pan/tilt mirror. Figure 2.1 shows a current prototype of the MSDP that employs a 1200 lumens LCD projector, which has enough brightness and contrast to project images that can be seen in a room with the lights on.

As illustrated in Figure 1, when the projection is not orthogonal to the surface the projected image can appear distorted. To correct the distortions caused by oblique projection, the image to be projected must be inversely distorted prior to projection. To accomplish this, our system first texture-maps the image onto a virtual computer graphics 3D surface. This surface is positioned in a 3D virtual space and a computer graphics board is used to render a pre-determined view of the surface that corrects the distortion. Of course, the appropriate position of the virtual 3D surface must be uniquely determined for each surface where images are projected.

\section{MAKING THE MSDP INTERACTIVE}

Although a device that simply projects images on multiple surfaces has many applications, we are particularly interested in making the projected surface interactive like a touch screen. This interactive enhancement of a MSDP is called a multi-surface interactive display projector, or MSIDP.

We are currently employing a pan/tilt video camera that is directed by the computer so it has a complete view of the projected surface. The user can interact by moving her hand

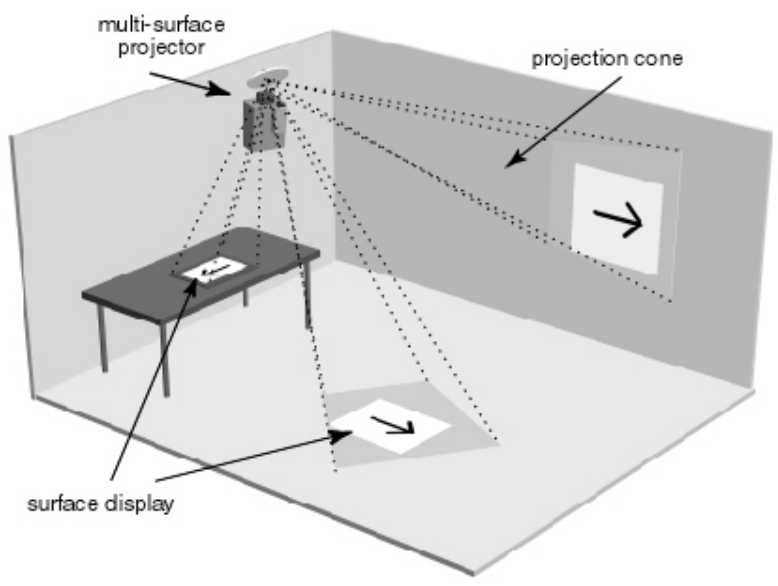

Figure 1. Using the multi-surface display projector to create displays on different surfaces in a room. 

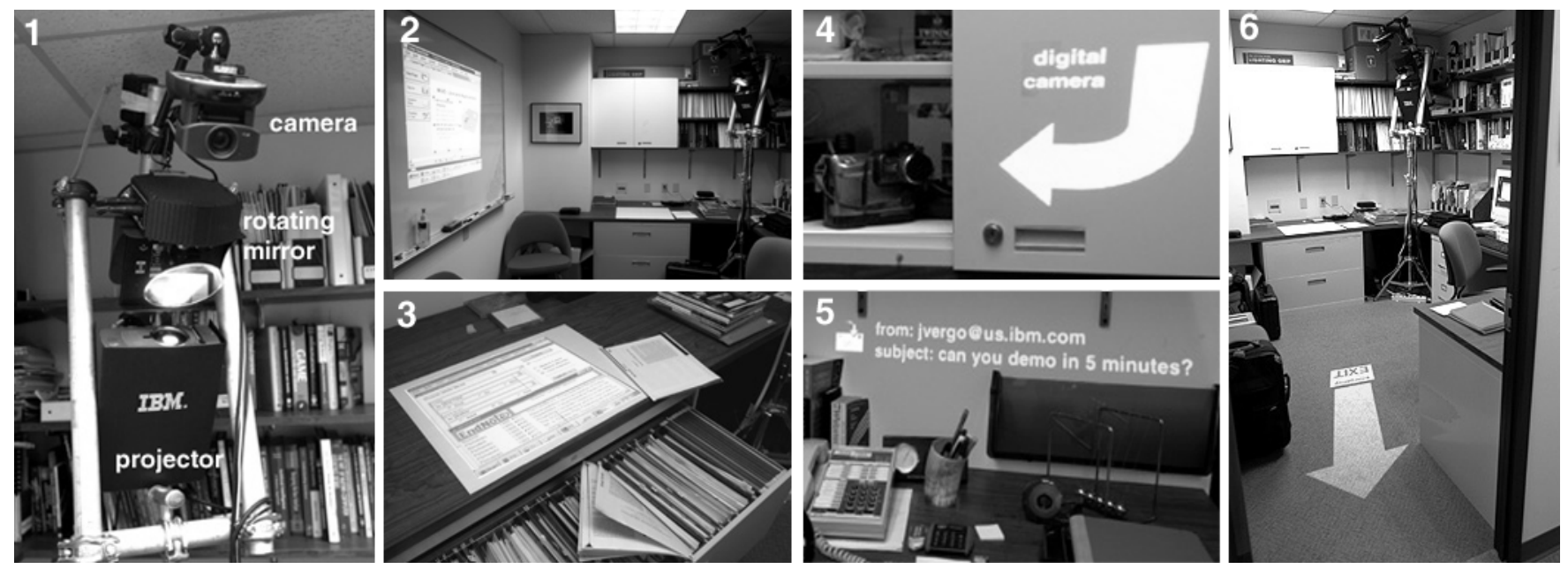

Figure 2. A prototype of the multi-surface interactive display projector (1) and examples of five different applications (2-6).

over the projected surface, as if the hand was a computer mouse; and by moving the hand rapidly towards the surface, to generate a "click" event. Techniques similar to the ones described in [1] are used to detect the hand's position and the interaction gestures.

\section{EXAMPLES OF APPLICATIONS}

The MSIDP is a generic input/output device with multiple applications. We are just beginning to explore such applications, as illustrated in the examples show in Figure 2.2-6.

A first class of applications corresponds to the creation of computer desktop-like interactive displays on non-tethered surfaces. Figure 2.2 shows a desktop application being projected on a whiteboard. Unlike the interactive whiteboard described in [1], the projected application can be moved around the room; for instance, to the top of a desk for more detailed reading, or to a wall if the area of the whiteboard is needed for writing or scribbling.

Similarly, the MSIDP can be used to bring information to the physical location where it is used or needed. Figure 2.3 shows a situation where a database application accessing a list of files is projected on the top of the file cabinet that contains those files. When looking for a particular file, the user can access and/or update information about the file.

A second class of applications deals with the provision of means for the computer to act on the physical world, almost like a robotic arm made of light. These applications can use the MSIDP to point to physical objects, show connections among them, and project dynamic patterns to indicate movement or change in the real world.

Figure 2.4 shows an example where the MSIDP responds a request for the position of an object (a digital camera) by directly pointing to the location of the object in the room. Figure 2.5 shows another example of spatial interaction where the MSIDP is used to project a notification of an email arrival on the wall. In Figure 2.6 the projector is used to signal the direction of an emergency exit. Notice that the rotating mirror allows one single MSIDP to switch among any of these different tasks as needed.

The MSIDP can also be used to provide computer and information access in spaces where traditional displays can be broken or stolen, or create hazardous conditions, such as in public spaces and areas subject to harsh environmental conditions. The device also permits an interactive display to be brought to the proximity of a user without requiring the user to move. In particular, the MSIDP can facilitate the access and use of computers by people with locomotive disabilities. For instance, it can project an interactive display on the sheet of a hospital bed without creating the risk of patient contact with any device.

\section{DISCUSSION}

Although there has been a substantial amount of research on interactive projected surfaces $[1,3,4]$, the concept of the MSIDP is unique in its combination of steerable projection and non-tethered display and interface. In particular, unlike tangible object-based interfaces [2], it does not require the construction or use of special, electronically enhanced objects. Instead, the MSIDP is a way to transform any surface or object, without modifications, into a generic data I/O device by simply projecting and sensing light.

\section{REFERENCES}

1. Crowley, J.L. et al. Things that See. Communications of the ACM, 43(3): 54-64. 2000.

2. Ishii, H. and B. Ullmer. Tangible Bits: Towards Seamless Interfaces between People, Bits, and Atoms. In Proc. of CHI'97. Atlanta, Georgia. 1997.

3. Raskar, R. et al. The Office of the Future: A Unified Approach to Image-Based Modeling and Spatially Immersive Displays. In Proc. of SIGGRAPH'98. Orlando, Florida. 1998.

4. Underkoffler, J. et al. Emancipated Pixels: Real-World Graphics in the Luminous Room. In Proc. of SIGGRAPH'99. Los Angeles, California. 1999. 